\title{
SIMULATION-BASED OPERATION MANAGEMENT OF OUTPATIENT DEPARTMENTS IN UNIVERSITY HOSPITALS
}

\author{
Byoung K. Choi \\ Donghun Kang \\ Joohoe Kong \\ Hyeonsik Kim \\ Industrial and Systems Engineering \\ KAIST, Daejeon, 305-701 \\ REPUBLIC OF KOREA
}

\author{
Arwa A. Jamjoom \\ Aisha M. Mogbil \\ Thoria A. Alghamdi \\ Faculty of Computing and Information Technology \\ King Abdulaziz University, Jeddah \\ KINGDOM OF SAUDI ARABIA
}

\begin{abstract}
Recently, the growth of outpatient clinic capacity has not matched the increasing demand on outpatient clinics, which has led to long waiting times for patients and overtime work for clinic staff. This has three significant negative effects on patients and staff: (1) patients' distrust of the procedures for treating outpatients increases, (2) nurses' stress from patient complaints increases, and (3) doctors' pressure to shorten treatment times while maintaining high levels of service quality increases. Presented in this paper is a simulation-based operation management method that provides the stakeholders with future visibility in outpatient departments. The future visibility is obtained from the current situation of the outpatient department using a simulation-based scheduling system and is shared by a business process management system that informs patients of their expected waiting time in order to lower the workload and pressure on clinic staff and to allow staff to manage exceptions proactively.
\end{abstract}

\section{INTRODUCTION AND LITERATURE REVIEW}

King Abdulaziz University (KAU) Hospital is one of the largest university hospitals in the Kingdom of Saudi Arabia. In 2011, KAU Hospital had a capacity of approximately 900 beds, and it was divided into two sections (public and private sections) that serve large number of patients. A university hospital such as KAU Hospital aims to maximize the quality of service by utilizing the limited resources to their maximum capacity. Specifically, the outpatient departments focus on improving the quality of service regarding the waiting time and congestion. Over the years, unreasonably long waiting times in outpatient clinics have been the focus of research among academicians and practitioners (McCarthy, McGee, and O'Boyle 2000; Zhu, Heng, and Teow 2012).

According to Günal and Pidd (2010), the waiting time in outpatient clinics can be classified into the $m$ icro waiting time of when a patient arrives at the clinic and the wait until a doctor calls them for treatment and the macro waiting time of the delay between the referral date of when a patient needs specialist treatm ent and the actual date of the specialist treatment. The macro waiting time tends to be longer because the growth of outpatient clinic capacity cannot match the increasing demand (Zhu, Heng, and Teow 2012). In KAU Hospital, the overbooked time slots are arranged to mitigate long macro waiting times; however, this system leads to increased workloads for outpatient clinic sessions. Moreover, the increased number of walk-in patients aggravates the workload on the clinic staff, which results in the already overloaded sessio $\mathrm{n}$ becoming more congested and patients must wait longer for their consultation. Despite this, overtime se ssions are becoming increasingly common and even normal.

The long waiting time and high congestion have negative effects on both patients and staff: (1) patients' distrust of the procedures for treating outpatients increases, (2) nurses' stress from patient com 
plaints increases, and (3) doctors' pressure to shorten treatment times while maintaining high levels of service quality increases (Rohleder et al. 2011; Zhu, Heng, and Teow 2012). Furthermore, for obstetric patients, it is a very undesirable situation to wait long times in a congested waiting room without knowing when they will see a doctor.

Recently, the concept of a "Smart Hospital" has been presented as a key to continuously improving the quality of service in order to maximize patient safety and customer service in university hospitals and large-scale hospitals (Fuhrer and Guinard 2006; Intelligent InSites 2013). According to Fuhrer and Guinard (2006), radio frequency identification (RFID) technologies can be used to build smart hospitals that optimize business processes, reduce errors, and enhance the quality of service. Also, real time locating systems (RTLS) have been adopted in order to locate patients, personnel, and equipment instantly, which ensures that the required equipment and personnel are located immediately and decreases the time spent on assigning tasks and checking their status (Intelligent InSites 2013).

These approaches to the smart hospital concept have something in common in that they are using advanced technologies to obtain the tractability in hospital operations, which is the ability to know what is happening in the hospital in real time. With tractability, however, the micro waiting time still remains invisible to patients (Pope 1993), and this might increase distrust and lack of understanding of the scheduling system (Lacy et al. 2004). Therefore, the staff who are in these delayed or uncertain settings might be pressured to respond to patient complaints and monitor their waiting times. Moreover, emergency requests may require rescheduling of patient appointments, which is not a simple job and involves communication with a number of dissatisfied patients (Fomundam 2007).

Presented in this paper is a simulation-based operation management that offers a new concept of outpatient operation in university hospitals. The proposed methodology provides the stakeholders (outpatients, nurses, residents, interns, and consultants) with the future visibility of the outpatient department. The future visibility is obtained using the current situation of the outpatient department and the simulation-based scheduling system, and it is shared using workflow technology, which may lead to increasing visibility of the waiting times for the patients, to lowering workload and pressure on clinic staff that arises from patient complaints, and to allowing staff to manage exceptions proactively.

Simulation-based operation management is not a new concept; in high-tech companies that produce LCDs, semiconductors and so on, the on-time delivery, which is one of the critical measures for the customer satisfaction, depends on the scheduling of activities, resources, and material flow (Part et al. 2008; Heilal et al. 2010). Old static production planning methods including mathematical equations and heuristic rules are not adequate because the production planners need accurate and dynamic models of production, which can be achieved by a simulation model that uses a production network and real shop flow data in real time (Heilala et al. 2010).

Recently, the application of a simulation has evolved into the day-to-day operational production and planning of manufacturing facilities from the off-line analysis of evaluating layout and dispatching rules (Park et al. 2008; Heilala et al. 2010; Frantzéna, Ng, and Moore 2011). In the day-to-day operational production, the online simulation is used, which has connections with real systems that store the current status of the manufacturing facilities (Hanisch, Tolujew, and Schulze 2005). The online simulation provides the stakeholders with the ability to evaluate the capacity of the facility for new orders, unforeseen events, expected delivery time, and changes in operations (Heilala et al. 2010). In Park et al. (2008), the concept of a simulation-based operation management is realized for LCD fabrication lines, which adopts an online simulation to obtain the future visibility of the factory operation and shares this information with the various stakeholders involved. This leads to improvements in the performance measures of the factory operation and shortened decision making cycles with reduced communication errors.

The workflow technology provides a software system, i.e. a workflow management system (WfMS), that thoroughly defines and automatically executes workflows in order to manage the actual flow of work so that the right work is done at the right time with the right information by the right person in the organization (WfMC 1995). Also, the application of this technology can be extended to the development of a reconfigurable information system that enables rapid implementation and maintenance (Lee and Choi 2011). In this paper, the workflow technology is not limited to the sharing of the future visibility; but also 
used for developing the outpatient operation management system that can be extended to work for other configurations of outpatient departments.

The remainder of this paper is organized as follows. Section 2 provides an overview of the obstetricsgynecology (OB-GYN) department of KAU Hospital, which is the target location for the implementation of the prototype system . Section 3 presents the business architecture of outpatient operation management. Section 4 describes the major functions of the proposed simulation-based operation management system. Section 5 provides an illustrative example of some functions under development. Conclusions and discussions are given in the final section.

\section{OB-GYN DEPARTMENT OF KING ABDULAZIZ UNIVERSITY HOSPITAL}

The OB-GYN outpatient department at KAU Hospital, which provides obstetric and gynecologic care to the public, is one of most congested departments and it has longer waiting times than other outpatient departments at KAU Hospital. The OB-GYN outpatient department has a total of 21 clinics that operate at different times throughout the day and night. Each clinic is operated by a consultant and a team of interns, residents, and nurses. Each day, the assigned number of clinics varies from 2 to 6 , and the number of clinics available per day may change.

The operation of the OB-GYN outpatient department for one session is separated into three phases: preparation, service, and wrap-up. In the preparation phase, the consultants whose clinics will be opened in the upcoming session meet in order to allocate the available residents to a consultant's team based on the assigned appointments and to distribute patients to each team. This phase starts at least 30 minutes prior to the session starting and ends before the session starts.

In the service phase, the consultants and their team provide outpatient services to the patients during the official session hours. Once the session starts, each patient goes through several pathways that vary according to the patient type (e.g. new or follow-up, booked or walk-in). The details of the patient flows are as follows. Upon arrival at the department's reception, a patient registers. If the patient is a "walk-in", she will wait until her patient file is brought to the department. The walk-in patient waits in the waiting room until she is called for a basic exam by the screening nurse. The screening nurse records the patient's weight and blood pressure. The patient returns to the waiting room and waits for the doctor. If the patient is "new", she must visit the intern first for an interview-based medical examination (check), then she moves to the screening nurse. When she is called by the doctor, she will see either the resident or a consultant for the consultation. The patient will book her next visit based on the doctor's assessment or leave the clinic if no further examination or appointment booking is required. The receptionist manages the booking processes for the patients. If the patient fails to reserve a regularly scheduled time slot for the requested date, she will visit the appointment management office in order to reserve an overbooked time slot. If the doctor requests for a blood test or X-ray, the patient visits the lab or X-ray department. The patient can have a blood test or exam on the same day of the current visit or can make an appointment on the same day of the next visit, prior to the consultation.

In the wrap-up phase, when it reaches the regular closing time for a session, the consultants in the session leave their consultation room for the remainder of the day. The residents will treat all not-yettreated patients that registered during the regular session hours using overtime hours.

\section{FRAMEWORK OF SIMULATION-BASED OUTPATIENT OPERATION MANAGEMENT}

Figure 1 presents the overall business architecture of the proposed outpatient operation management system that covers the three phases of the OB-GYN outpatient department at KAU Hospital. In addition, one more phase ( $D$-scheduling phase) is introduced in order to minimize the variability that is inherent in patient arrival. The four scheduling workflows are defined for each phase of the outpatient operation and are managed by the business process management (BPM) system that will be explained in Section 4 .

The $D$-scheduling workflow coordinates patient scheduling using patient appointments and initiates $x$ days prior to the target session. The objective of this workflow is to generate the patient schedule for the booked patients who will visit the clinic on $D-0$ day and to obtain the confirmations from the booked pa- 
tients. Through completing this, the booked patients will be provided with their scheduled appointment time to visit the clinic and the prospect of non-attendance will be reduced.

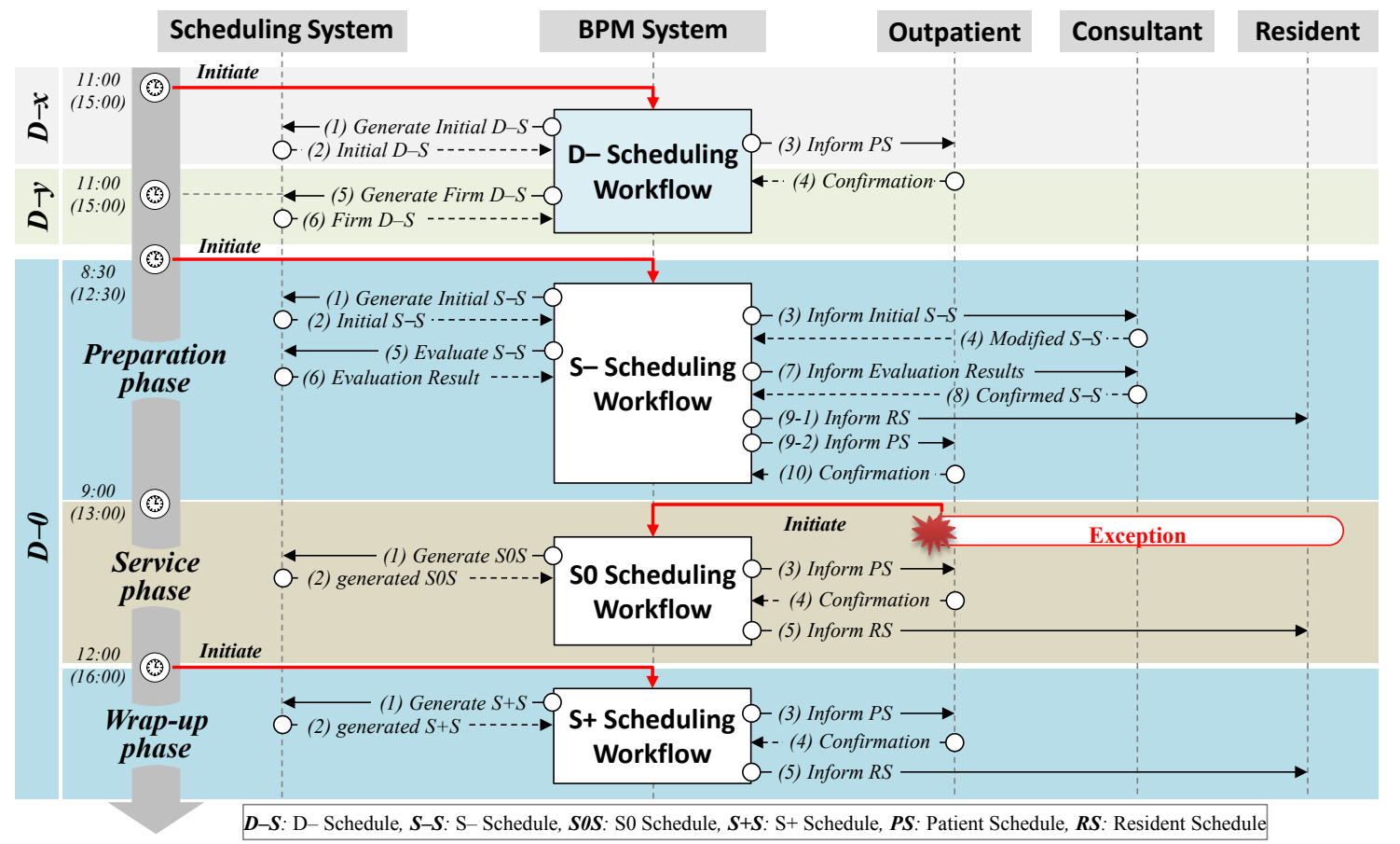

Figure 1: Overall business architecture of simulation-based outpatient operation management

In order to achieve the objective, it requests the scheduling system to generate an initial D- schedule (D-S), which is the initial version of the patient schedule (PS) that specifies the appointment time of each booked patient in the target session. The initial D-S contains performance measures that are evaluated using the simulation. Then, the D- scheduling workflow distributes the patient schedule to the respective patients who have booked an appointment in the target session. Finally, the received patient schedule will be confirmed or canceled by the patient. As shown in Figure 2, once the patient schedule is distributed, $\alpha$ (12 or 24) hours later, the scheduling system will generate the firm D-S by reflecting the responses from the patients. The firm D-S contains the fixed times of patient appointments confirmed by the booked patients and non-responded appointments will be canceled.

The $S$ - scheduling workflow supports the preparation phase of the outpatient operation and it starts 30 minutes before the target session starts. The objective of this workflow is to assist the consultant in allocating the residents to each consultant team and to create the firm $S$-schedule $(\mathrm{S}-\mathrm{S})$ with regard to the changes in the patient appointments and resource absences that occurred after the firm D-S was created. In doing so, the consultant will be provided with the quantitative performance measures for the $\mathrm{S}-\mathrm{S}$, which will facilitate the decision making process with a better quality of service.

As depicted in Figure 3, firstly, it requests that the scheduling system generates the initial S- schedule (S-S) that includes the resident schedule (RS), which contains data about the residents allocation to each consultant and patient schedule. The initial S-S will be verified by the head nurse and then delivered to the consultants. The consultants review the initial S-S with the support of the simulation service provided by the scheduling system. Quantitative evaluations via the simulation can test the alternatives of the Sschedules with respect to the quality of service, such as waiting times and congestion. Once the $\mathrm{S}-\mathrm{S}$ is reviewed by the consultant, it will be distributed to the residents, head nurse, and scheduled patients. 
Choi, Kang, Kong, Kim, Jamjoom, Mogbil, and Alghamdi

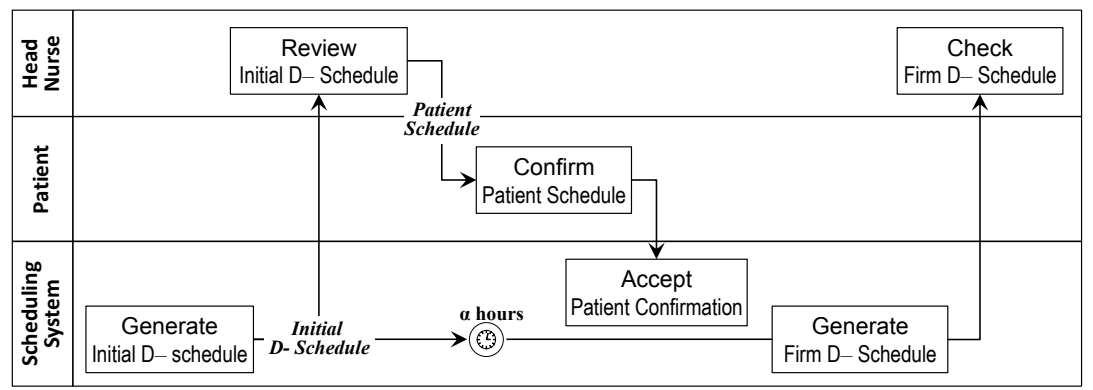

Figure 2: Swim-lane chart of D- scheduling workflow

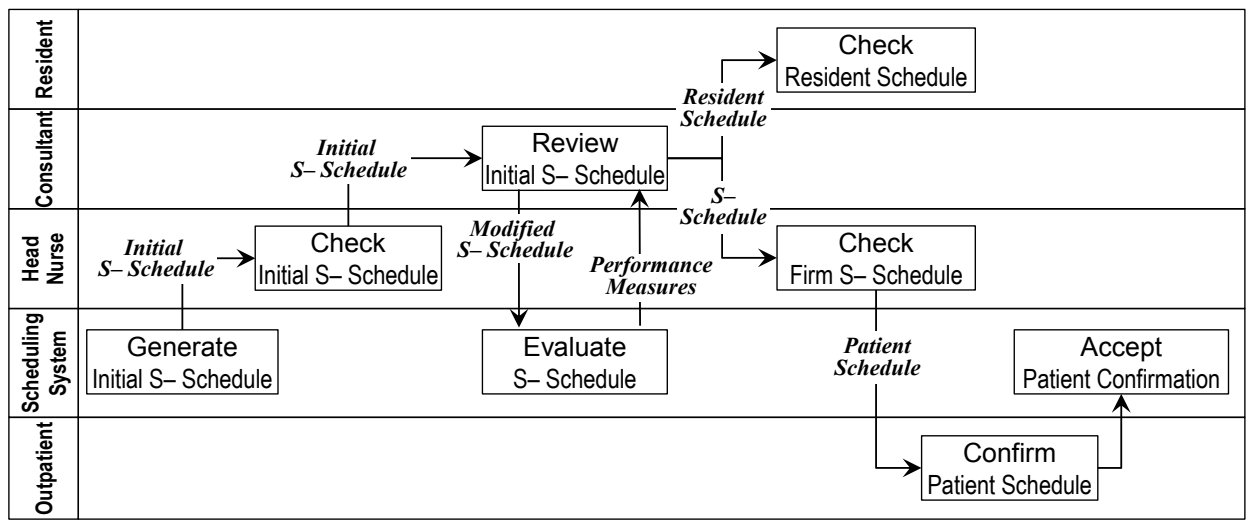

Figure 3: Swim-lane chart of S- scheduling workflow

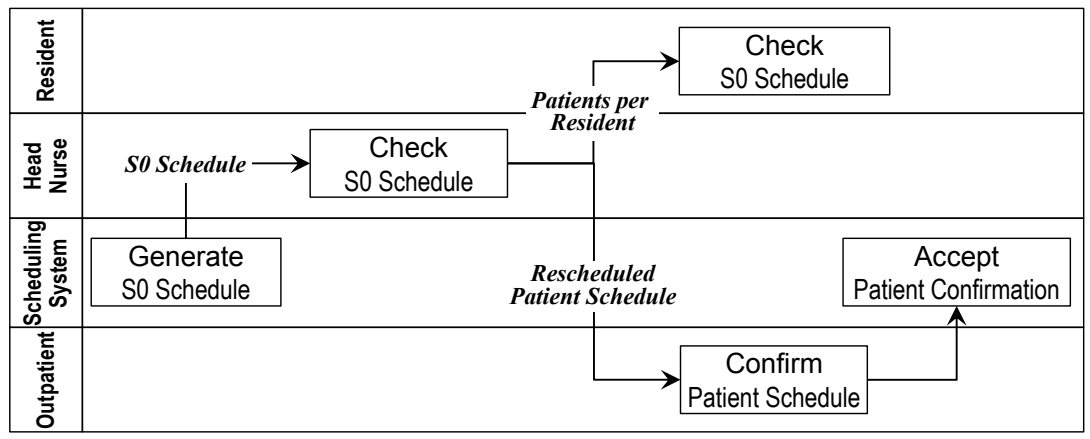

Figure 4: Swim-lane chart of S0 scheduling workflow

The SO Scheduling Workflow handles the exceptions during the service phase of the outpatient operation, such as emergency call for consultants, absence of consultants, or high congestion in the waiting room. Figure 4 shows the swim-lane chart of S0 Scheduling Workflow, which is designed to mitigate the difficulties in rescheduling patient appointments and communicating with patients. Once an exception occurs, the head nurse may initiate the S0 scheduling workflow. The scheduling system generates a S0 schedule (SOS), which reallocates the residents to take over the remained patients. The head nurse checks whether the SOS is valid or not; then, the SOS will be distributed to the residents and patients. The scheduled patients will receive a message stating that the resident has been reassigned and the time of the rescheduled appointment including an expected waiting time. If the patient cannot wait for the rescheduled appointment, she will notify this to the scheduling system or head nurse directly.

The $S+$ Scheduling Workflow support the wrap-up phase of the outpatient operation by rescheduling the patient schedule in order to minimize the waiting time and closing time of a session. The behavior of 
the $\mathrm{S}+$ scheduling workflow is the same as that of the S0 scheduling workflow. From this workflow, each patient will know when their consultation is scheduled and how much time they are required to wait.

The four scheduling workflows make it easy communicate with patients by letting them know the expected appointment time before the session and expected waiting time in the session. Also, the workflows provides the future visibility that helps the decision making process of staffs to act against the exceptional situations (e.g. doctor's emergent absence or accepting more walk-in patients).

\section{FUNCTIONS OF SIMULATION-BASED OUTPATIENT OPERATION MANAGEMENT SYSTEM}

In this section, the system structure of the simulation-based outpatient operation management is described. Figure 5 presents our proposed system divided into a BPM system and a simulation-based scheduling system. The BPM system orchestrates the execution of simulation-based outpatient operation by managing the four scheduling workflows described in Section 3. In each scheduling workflow, the modules of the simulation-based scheduling system are invoked to provide the generation of the resident schedule and patient schedule and the evaluation of the generated schedules.

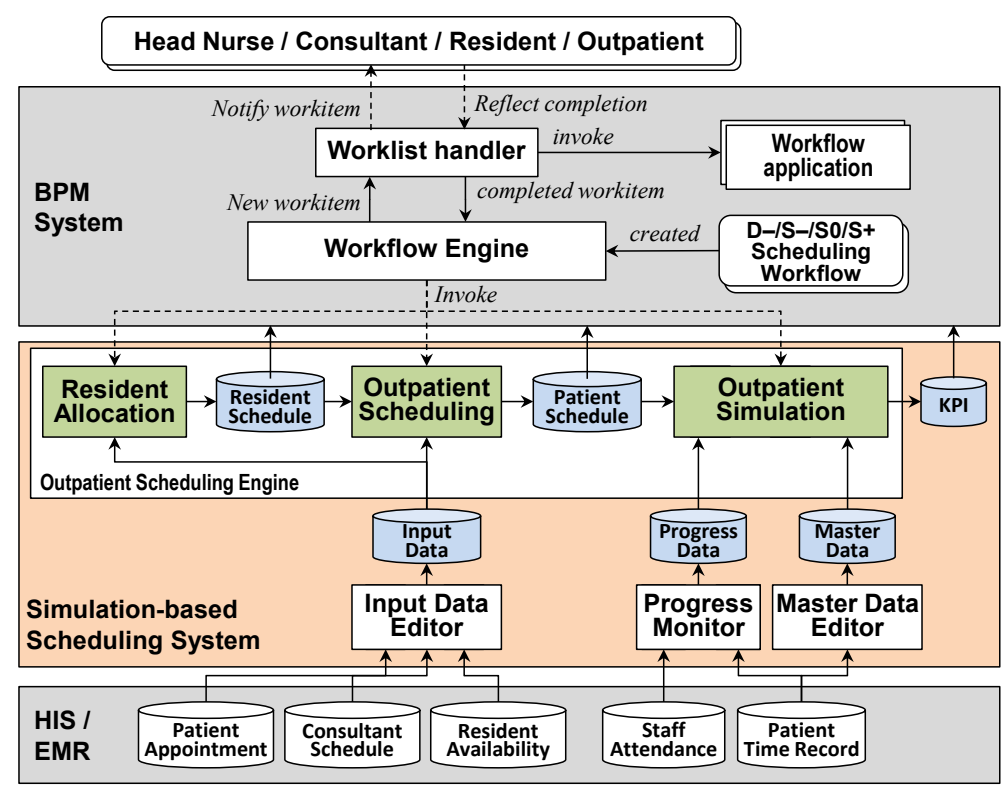

Figure 5: System architecture of simulation-based outpatient operation management system

\subsection{Simulation-based Scheduling System}

The core part of the simulation-based scheduling system is the outpatient scheduling engine that performs the resident allocation, generates patient schedule, and evaluates the performance of a session with a given resident schedule and a patient schedule. Each module of the outpatient scheduling engine can be executed as a standalone by the invocation of the respective scheduling workflow. The Resident Allocation module takes a resident availability, a consultant schedule, and a patient appointment data, and it computes the resident schedule of which resident is allocated to a consultant's team. The Outpatient Scheduling module takes a consultant schedule, a patient appointment, and a resident schedule, and it computes the patient schedule of when a booked patient will be treated and by which consultant. The algorithm of generating a patient schedule and a resident schedule can be differentiated for each configuration of the outpatient department, and it can be borrowed by existing appointment scheduling schemes (Soriano 1966; Smith, Schroer, and Shannon 1979; Rohleder and Klassen 2002).

The Outpatient Simulation module provides the online simulation of the patient flows for a session from the current situation of an outpatient department. Many studies have been focused on the use of off- 
line simulation for evaluating various scheduling schemes and operational rules (Smith, Schroer, and Shannon 1979; Rohleder and Klassen 2002; Guo, Wagner, and West 2004; Rohleder et al. 2011; Zhu, Heng, and Teow 2012). According to Günal and Pidd (2010), however, each study focused on a specific case or a configuration in an ad-hoc way that cannot be generalized and applied to other situations or environments. Also, the commercial simulation packages such as Arena ${ }^{\circledR}$ and Simul ${ }^{\circledR}$ are not possible to develop a reusable problem solving tool for the outpatient scheduling.

To mitigate this situation, we have constructed a formal discrete-event simulation model, which provides a general representation of any outpatient department (Choi et al. 2013b). The constructed simulation model regards the patient flows of the outpatient clinics as a job shop, which is characterized by a number of stations with each station having one or more identical machines and multiple job types with each job type having a unique routing sequence. The complex behavior of patient flows, which can be differentiated by each doctor and each patient type, are represented using the Parameterized Activity Cycle Diagram (P-ACD) model of a job shop. The P-ACD is an extension of activity cycle diagram (ACD) with the parameterization, which can model the job shop (Choi et al. 2013a).

With the formal simulation model, the user can easily adapt the simulation to his/her situation without constructing a new simulation model or modifying existing simulation model. The only thing that has to be done is updating master data and progress data. Additional software modules help the users update these two data. Master data editor is responsible for maintaining correct master data which is one of the essential tasks in this type of system. Master data includes (1) Bill of Process (BOP) data for each patient type, a sequence of activities a patient go through in a clinic, (2) Resource capacity data for each staff and physical resource, (3) Resource assignment data of relationship between activities and resources with processing times, (4) Patient type ratio data for each patient type, a ratio of which patient type will be a patient, and (5) Patient arrival data of the amount of time that the patient will be arrived earlier or later for the appointment, the inter-arrival time of walk-in patients, and no-show rate of booked patient.

Progress monitor receives the current status and progress of the outpatient department from the hospital information system (HIS) or electronic medical record (EMR) system, and it computes up-to-date progress data. The progress data includes the patient status of where the patient waits or has a consultation and staff status of absence or working. Another use of progress monitor is to compare the actual progresses against the planned targets in the patient schedule. If any abnormal delay is observed, it notifies a head nurse or other staff by sending a warning message.

\subsection{Business Process Management System}

According to the Workflow Management Coalition (WfMC 1999), a workflow is a collection of tasks organized to accomplish a business process executable by workflow engine that is a major component of WfMS. The instance of a workflow is created by the workflow engine when the start condition of the workflow satisfied. Then, the tasks in the workflow are arranged to execute by sending out its work items to the respective participant via worklist handler. The worklist handler is a software component that manages the interaction between the participants and the list of work items maintained by the workflow engine and invokes workflow applications if needed to support the processing of a work item.

Here, the invoked workflow application may automate a task, fully or in part, or to support a participant in processing a task and it can be a form of a web service, or a server application, a client application, or a web application, or other form of executable software modules. In our case, the modules of simulation-based scheduling system are part of workflow applications and additional workflow applications are provided to support the processing of tasks. Please, refers to the following section for more details.

In recent years, WfMS has evolved into business process management (BPM) system by supplementing it with several new functionalities (Delphi 2003). The commercial BPM systems provide the modeling capability of the workflows. In the workflow modeling, business process model and notation (BPMN) is widely accepted and supported in the BPM system because of it is readily understandable by all business users from the business analysts to technical developers (OMG 2011). Figure 6 shows the BPMN 
representation of D- Scheduling Workflow of Figure 2. Also, the rest of the scheduling workflows can be represented using BPMN, but they are omitted for space constraint condition.

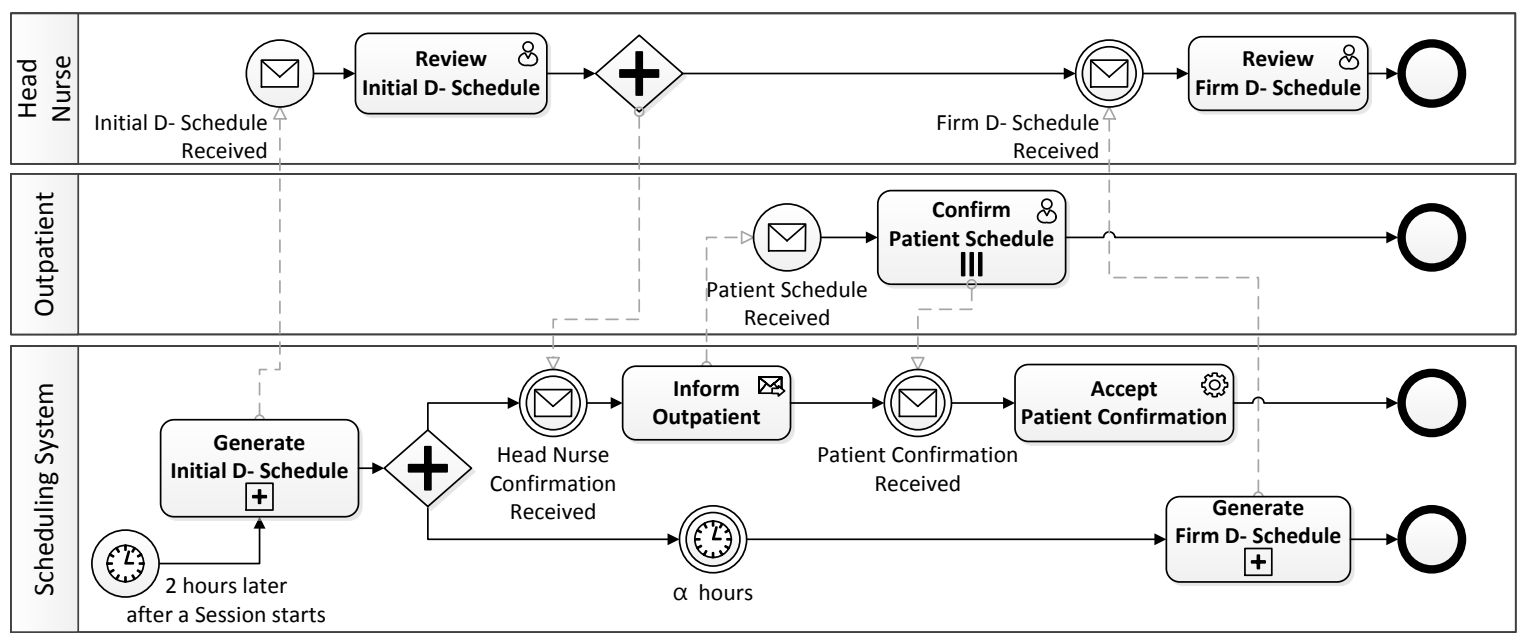

Figure 6: BPMN model of D- Scheduling Workflow

\section{ILLUSTRATIVE EXAMPLE}

A prototype system of the simulation-based operation management depicted in Section 3 that follows the system architecture presented in Section 4 is under the development. In the proof-of-concept implementation, an academic BPM system (Choi and Hwang 2005), which are in compliance with the WfMC standards (WfMC 1998), has been deployed to manage the four scheduling workflows presented in Section 3.

All the modules of the simulation-based scheduling system presented in Section 4 are implemented in $\mathrm{CA}$ programming language. Especially, the outpatient simulation module has been developed in the authors' previous work (Choi et al. 2013b) using a formal modeling method, which are ready for a different configuration of an outpatient departments using the input data and master editors. Using the two editors, we have provided a configuration for our case which will be explained in the following sub-sections.

\subsection{OB-GYN Department at KAU Hospital}

We have made a test case based on the observed data from OB-GYN outpatient department at KAU Hospital. For a D- Scheduling Workflow, we assume that a target session has three clinics. Each clinic is operated by a consultant and a number of residents. Five residents are available for the target session. The D- Scheduling Workflow are initiated three days before the target session. Initially, there are 148 booked patients over the three clinics. Each patient may be new to the clinic or may revisit the clinic. The patient appointment, the consultant schedule, and the resident availability are collected using the input data editor. The master data including the BOP data found in Section 2 are defined and managed via the master data editor as shown in Figure 7.

\subsection{D- Scheduling Workflow}

Figure 8 shows the process definition models (PDMs) that implements the BPMN model of $D$ - Scheduling Workflow depicted in Figure 6. In the WfMS, the concept of a PDM is used to represent all the necessary information about the process to enable it to be executed by the WfMS (WfMC 1998). The PDM of the $D$-Scheduling Workflow has two sub processes, named as Generate Initial D-Schedule and Generate Firm D-Schedule. The sub process is a process that is enacted or called from another process and which forms part of the overall process (WfMC 1999). Each sub process carries out the constitute agent-type activities to compute the initial and firm D- schedules by invoking the respective workflow applications, such as the modules of the simulation-based scheduling system as depicted in Figure 5. 
Choi, Kang, Kong, Kim, Jamjoom, Mogbil, and Alghamdi

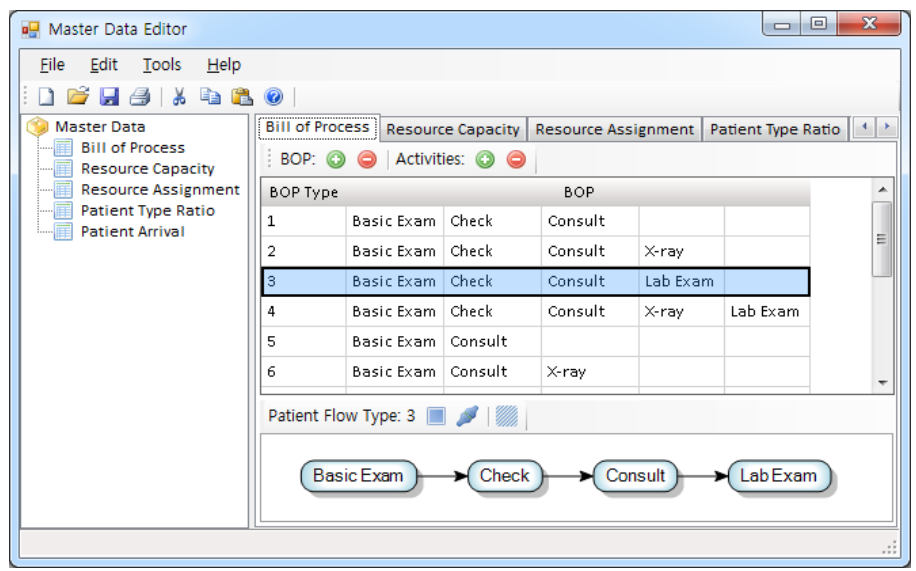

Figure 7: Master data editor with a BOP data

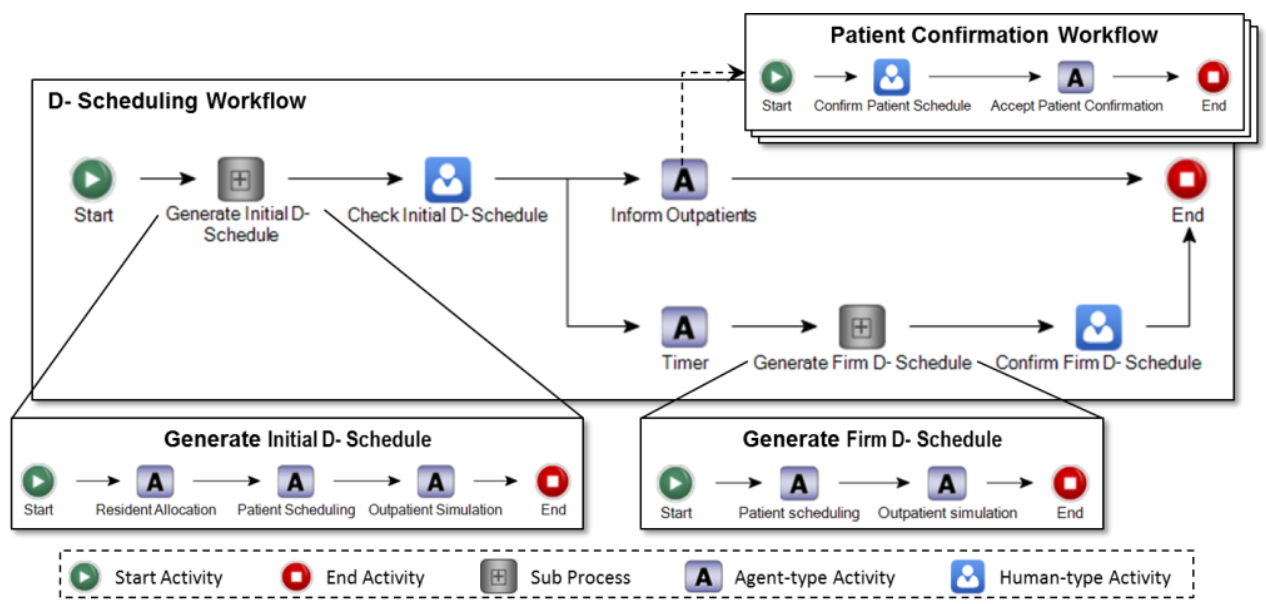

Figure 8: Process Definition Models of D- Scheduling Workflow in Figure 6

At the Inform Outpatients activity, the Patient Confirmation Workflow PDM is initiated for each patient listed in the patient schedule. As the Patient Confirmation Workflow PDM starts, each patient receives a message through a short message service (SMS) and replies with a simple answer, such as 1, 2, or a specific time. Upon the receipt of the returned SMS message, the patient confirmation in the message will be reflected in the patient schedule. These PDMs have been modeled with a Process Designer module of the BPM system.

An activity that requires human is called human-type activity, such as Check Initial D-Schedule, Confirm Firm D-Schedule, and Confirm Patient Schedule. This type of activities may need an invoked application to help the participant perform the tasks. Especially, Check Initial D-Schedule and Confirm $D$-Schedule activities, which require a head nurse to review the generated D- schedule, have an invoked application that shows the D- schedule to a head nurse.

Figure 9 shows the invoked application for Check Initial $D$-Schedule activity that presents the initial D- schedule of the target session. The calendar view shows the patient schedule of a specific clinic and the table located at the upper shows the residents allocated to each clinic, number of booked patients, expected close time of the target session with the number of booked patients, remaining time to the regular close time of the target session. Other scheduling workflows defined in Section 3 (S-, S0, and S+ scheduling workflow) have also been implemented as the $D$-Scheduling Workflow has been done. 
Choi, Kang, Kong, Kim, Jamjoom, Mogbil, and Alghamdi

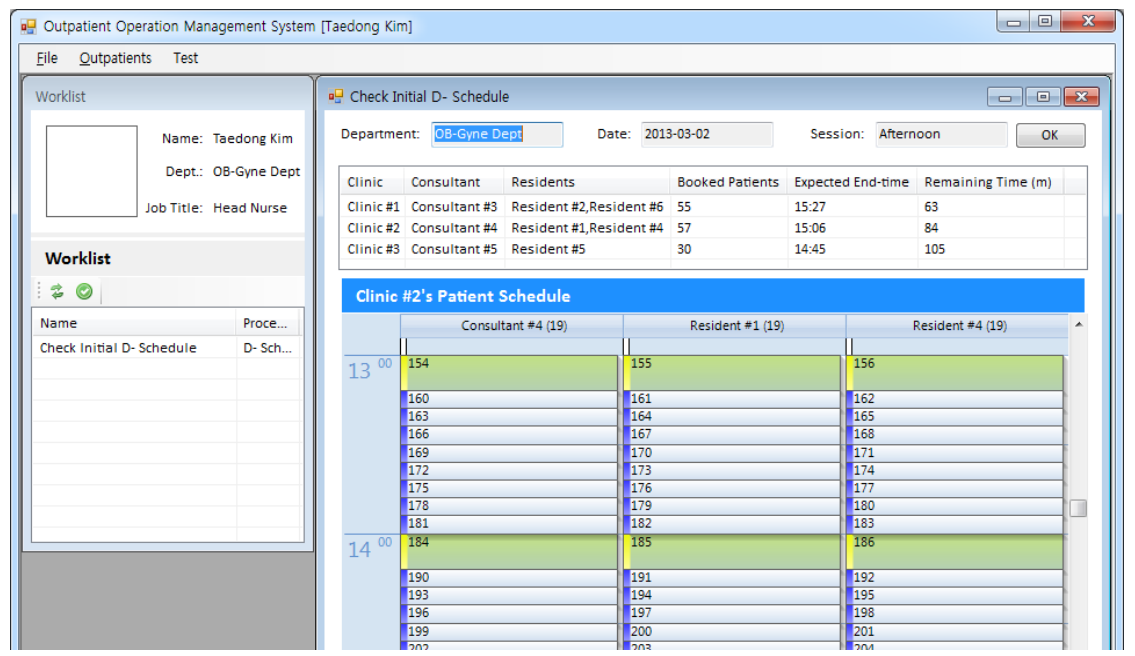

Figure 9: Invoked application for "Check Initial D- Schedule" activity

\section{CONCLUSION}

In this paper, we presented a novel approach, simulation-based operation management, for the outpatient operation of outpatient departments at university hospitals. It consists of simulation-based scheduling system and BPM system. The BPM system defines and executes the proposed scheduling workflows that support the four phases of the outpatient operation. The simulation-based scheduling system provides the future visibility of the outpatient department in response to the invocation from the scheduling workflows through simulations constructed with a formal modeling method, P-ACD.

The simulation-based operation management promotes the sharing of the future visibility of the outpatient operation to the stakeholders, which may lead to reduce the invisibility of waiting time to the patients, to lower the workload and pressure on the clinic staff, and to allow the staffs to handle exceptions proactively. The proof-of-concept system for the simulation-based operation management is still under the development. In the near future, however, the feasibility study of the simulation-based operation management will be conducted as soon as the prototype system is available. As indicated in Park et al. (2008), the challenge that lies on maintaining valid master data and to acquire real-time progress data from the outpatient department will be investigated in further research.

\section{ACKNOWLEDGMENTS}

The research is partially supported by King Abdulaziz University (project title: Generalized-ACD for Modeling and Simulation of Complex Discrete-Event Systems) to which the authors are grateful.

\section{REFERENCES}

Choi, B. K. and H. C. Hwang. 2005. "Architecture of A 3-Layered Closed-loop BPMS Harmony (in Korean).” Technical Report, VMS Laboratory, KAIST. Accessed August 4. http://vms.kaist.ac.kr.

Choi, B. K, D. Kang, T. Lee, A. A. Jamjoom, and M. F. Abulkhair. 2013a. "Parameterized Activity Cycle Diagram and its Application." Transactions on Modeling and Computer Simulation, accepted.

Choi, B. K., J. Kong, H. Kim, D. Kang, A. A. Jamjoom, and A. M. Mogbil. 2013b. "Parameterized Formal Modeling of Outpatient Clinics in University Hospitals." Technical Report, VMS Laboratory, KAIST. Accessed August 4. http://vms.kaist.ac.kr.

Delphi Group. 2003. BPM 2003 Market Milestone Report. White Paper. Accessed August 4. http://www.delphigroup.com/whitepapers/pdf/20031023-BPM.pdf 
Frantzén, M., A. H. C Ng, and P. Moore. 2011. "A Simulation-based Scheduling System for Real-time Optimization and Decision Making Support." Robotics and Computer-Integrated Manufacturing 27:696-705.

Fuhrer, P. and D. Guinard. 2006. "Building a Smart Hospital using RFID technologies." In Proceedings of ECEH 06 (European Conference on eHealth), Fribourg, Switzerland, 131-142.

Guo, M., M. Wagner, and C. West. 2004. "Outpatient Clinic Scheduling: a Simulation Approach." In Proceedings of the 2004 Winter Simulation Conference, Edited by R .G. Ingalls, M. D. Rossetti, J. S. Smith, and B. A. Peters, 1981-1987. Piscataway, New Jersey: Institute of Electrical and Electronics Engineers, Inc.

Günal, M. M. and M. Pidd. 2010. "Discrete Event Simulation for Performance Modelling in Health Care: a Review of the Literature." Journal of Simulation 4:42-51.

Hanisch, A., J. Tolujew, and T. Schulze. 2005. "Initialization of Online Simulation Models." In Proceedings of the 2005 Winter Simulation Conference, Edited by M. E. Kuhl, N. M. Steiger, F. B. Armstrong, and J. A. Joines, 1795-1803. Piscataway, New Jersey: Institute of Electrical and Electronics Engineers, Inc.

Heilala, J., J. Montonen, P. Järvinen, S. Kivikunnas, M. Maantil, J. Sillanopää, and T. Jokinen. 2010. "Developing Simulation-based Decision Support Systems for Customer-driven Manufacturing Operation Planning." In Proceedings of the 2010 Winter Simulation Conference, Edited by B. Johansson, S. Jain, J. Montoya-Torres, J. Hugan, and E. Yücesan, 3363-3375. Piscataway, New Jersey: Institute of Electrical and Electronics Engineers, Inc.

Intelligent InSites. 2013. Enabling Process Improvements in Healthcare through Real Enterprise Visibility, White Paper. Accessed August 4. http://www.intelligentinsites.com.

Lacy, N. L., A. Paulman, M. D. Reuter, and B. Lovejoy. 2004. "Why We Don't Come: Patient Perceptions on No-Shows." The Annals of Family Medicine 2(6):541-545.

Lee, H. Y. and B. K. Choi. 2011. "Using Workflow for Reconfigurable Simulation-based Planning and Scheduling System." International Journal of Computer Integrated Manufacturing 24:171-187.

McCarthy, K., H. M. McGee, and C. A. O'Boyle. 2000. "Outpatient Clinic Waiting Times and NonAttendance as Indicators of Quality." Psychology, Health \& Medicine 5:287-293.

OMG (Object Management Group) 2011. "Business Process Model and Notation (BPMN) Version 2.0." Accessed April 10. http://www.omg.org/spec/BPMN/2.0.

Park, B. C., E. S. Park, B. K. Choi, B. H. Kim, and J. H. Lee. 2008. "Simulation based Planning and Scheduling System for TFT-LCD Fab." In Proceedings of the 2008 Winter Simulation Conference, Edited by S. J. Mason, R. R. Hill, L. Mönch, O. Rose, T. Jefferson, and J. W. Fowler, 2271-2276. Piscataway, New Jersey: Institute of Electrical and Electronics Engineers, Inc.

Pope, C. 1993. "Waiting Times for Outpatient Appointments." British Medical Journal 306(6875):408409.

Rohleder, T. R. and K. J. Klassen. 2002. "Rolling Horizon Appointment Scheduling: A Simulation Study." Health Care Management Science 5:201-209.

Rohleder, T. R., P. Lwekonia, D. P. Bischak, P. Duffy, and R. Hendijani. 2011. "Using Simulation Modeling to Improve Patient Flow at an Outpatient Orthopedic Clinic." Health Care Management Science 14:135-145.

Smith, S. R., B. J. Schroer, and R. E. Shannon. 1979. "Scheduling of Patients and Resources for Ambulatory Health Care." In Proceedings of the 1979 Winter Simulation Conference, Edited by H. J. Highland, M. G. Spiegel, and R. Shannon, 2:553-561. Piscataway, New Jersey: Institute of Electrical and Electronics Engineers, Inc.

Soriano, A. 1966. "Comparison of Two Scheduling Systems." Operations Research 14:388-397.

WfMC (Workflow Management Coalition) 1995. "The Workflow Reference Model.", WfMC-TC-001003. Accessed April 10. http://www.wfmc.org.

WfMC (Workflow Management Coalition) 1998. "Workflow Management Application Programming Interface Specification.” WfMC-TC-1009. Accessed April 10. http://www.wfmc.org. 
WfMC (Workflow Management Coalition) 1999. “Terminology \& Glossary.” WfMC-TC-1011. Accessed April 10. http://www.wfmc.org.

Zhu, Z., B. H. Heng, K. L. Teow. 2012. "Analysis of Factors Causing Long Patient Waiting Time and Clinic Overtime in Outpatient Clinics.” Journal of Medical Systems 36:707-713.

\section{AUTHOR BIOGRAPHIES}

BYOUNG K. CHOI is a professor of the Department of Industrial Engineering at KAIST in Daejeon, South Korea since 1983. He is also an adjunct professor at King Abdulaziz University in Jeddah, Saudi Arabia since 2012. His current research interests are system modeling and simulation, BPMS, simulationbased scheduling, and virtual manufacturing. His email address is bkchoi@kaist.ac.kr.

DONGHUN KANG is a postdoctoral researcher in the Department of Industrial and Systems Engineering at KAIST in Daejeon, South Korea. His research interests lie in the DES M\&S and its applications in various domains. His email and web addresses are donghun.kang@kaist.ac.kr and http://dhkang.org.

JOOHOE KONG is a Ph. D candidate in the Department of Industrial and Systems Engineering at KAIST in Daejeon, South Korea. Her research interests lie in the DES M\&S and its applications in areas of healthcare delivery and call center system. Her email address is joohoe.kong@vmslab.kaist.ac.kr.

HYEONSIK KIM is a Ph. D. candidate student in Department of Industrial and Systems Engineering in KAIST in Daejeon, South Korea. His current research area is DES M\&S. His email address is hyeonsik.kim@vmslab.kaist.ac.kr.

ARWA A. JAMJOOM is an assistant professor in the Department of Computer Science at King Abdulaziz University in Jeddah, Saudi Arabia. Her research interests lie in data warehousing and discreteevent simulation of healthcare delivery system. Her email address is ajamjoom@kau.edu.sa.

AISHA M. MOGBIL is a researcher in the Department of Information Systems at King Abdulaziz University in Jeddah, Saudi Arabia. Her interests lie in the medical information system and discrete-event simulation of healthcare delivery system. Her email address is a_megbil@yahoo.com.

THORIA A. ALGHAMDI is a researcher in the Department of Information Systems at King Abdulaziz University in Jeddah, Saudi Arabia. Her interests lie in managerial information system and discrete-event simulation of healthcare delivery system. Her email address is thalghamdi@yahoo.com. 